\title{
Fast Acquisition of Tilt-series for High-resolution Sub-tomogram Averaging
}

Alberto Bartesaghi ${ }^{1}$, Hsuan-Fu Liu ${ }^{1}$, Jonathan Bouvette ${ }^{2}$, Joshua Strauss ${ }^{3}$ and Mario Borgnia ${ }^{2}$

${ }^{1}$ Duke University, Durham, North Carolina, United States, ${ }^{2}$ National Institute of Environmental Health Sciences - NIH, Research Triangle Park, North Carolina, United States, ${ }^{3}$ University of North Carolina Chapel Hill, Chapel Hill, North Carolina, United States

Cryo-electron tomography (cryo-ET) combined with sub-volume averaging is a powerful imaging modality used to determine the structure of important biomedical targets within the native context of the cell. In order to obtain high-resolution structures, a large number of copies of the complex of interest need to be imaged so they can be aligned and averaged in three-dimensions using sub-tomogram averaging. Compared to single-particle cryo-EM, proteins imaged in-situ by cryo-ET are present at much lower concentrations requiring the collection of large amounts of data in order to accumulate enough particles to allow high-resolution structural analysis. Efforts to improve the quality of tomographic tilt-series resulted in the introduction of the dose-symmetric tilting-scheme which aims to maximize the amount of high-resolution information captured in the data by beginning at low tilts and alternating between increasingly positive and negative tilts [1]. The wait times incurred during tilt-series acquisition for tracking, autofocusing, and stage settling, however, make this approach slow and therefore incompatible with high-throughput imaging. More recently, strategies to speed up tomographic data collection have been proposed that take advantage of the capability of modern direct electron detectors to operate in movie mode. By continuously tilting the stage during the exposure or by stopping the stage at discrete tilt angles while recording the movie, these strategies can dramatically speed up data acquisition at the expense of reduced tracking and focusing accuracy [2]. Here, we explore the use of alternative strategies for tomographic data collection that are simultaneously compatible with high-throughput and high-resolution imaging. Our approach allows fast collection of large number of tilt-series with high tracking and focusing accuracy while also preserving the high-resolution information contained in the data. Availability of these strategies combined with hybrid data processing techniques for sub-tomogram averaging [3], will enable routine determination of high-resolution structures of protein complexes in their native state using cryoET.

\section{References}

[1] W. J. H. Hagen, W. Wan, and J. A. G. Briggs, "Implementation of a cryo-electron tomography tiltscheme optimized for high resolution subtomogram averaging," J. Struct. Biol., vol. 197, no. 2, pp. 191198, 2017.

[2] G. Chreifi, S. Chen, L. A. Metskas, M. Kaplan, and G. J. Jensen, "Rapid tilt-series acquisition for electron cryotomography," J. Struct. Biol., vol. 205, no. 2, pp. 163-169, 2019.

[[3] A. Bartesaghi, F. Lecumberry, G. Sapiro, and S. Subramaniam, "Protein secondary structure determination by constrained single-particle cryo-electron tomography," Structure, vol. 20, no. 12, pp. 2003-2013, 2012. 